\title{
Prendersi cura della generatività, genitorialità e cogenitorialità con gli operatori socio-sanitari per una profilassi psicoeducativa
}

L. Cena, A. Imbasciati

\subsection{Premessa}

In un nostro progetto di prevenzione perinatale tutt'ora in corso (capitolo. 11) per la tutela della salute mentale delle madri, dei padri e dei loro bambini è stata strutturata un'indagine esplorativa attraverso le principali aree tematiche relative alla perinatalità psichica genitoriale, evidenziate dalla letteratura. Alcune di queste aree verranno esaminate in questo e nel prossimo capitolo, in cui saranno presi in considerazione indicatori di rischio per la salute mentale perinatale, ma anche di protezione e promozione del benessere psicofisico della coppia e del bambino che tutti gli operatori socio-sanitari dei percorsi- nascita, attraverso l'assistenza alla pianificazione e realizzazione del progetto generativo e genitoriale, devono imparare a esplorare attraverso la loro funzione professionale ed educativa. Gli operatori dell'assistenza al percorsonascita (ostetriche, ginecologi, neonatologi, psicologi, assistenti sanitarie, assistenti sociali, puericultrici, infermieri, educatori) sono le figure professionali che a diversi livelli e con specifici ruoli sono coinvolte nel processo della perinatalità genitoriale e che possono operare, attraverso le loro funzioni e competenze, interventi orientati a un'assistenza sanitaria adeguata e a una profilassi psicoeducativa ${ }^{1}$.

Una formazione continua di tale operato si rivela pertanto opportuna a quanto è stato descritto come perinatalità psichica della coppia genitoriale, iniziando dai percorsi formativi universitari dei corsi di laurea triennale e specialistica, per proseguire con Master post-laurea, Corsi di perfezionamento, di aggiornamento (organizzati dall'Università) attraverso una formazione continua in medicina, i cosiddetti ECM. L'obiettivo con cui sono stati istituiti infatti gli ECM - crediti formativi - è proprio

\section{Cena (四)}

Professore Associato di Psicologia Clinica

Dipartimento Materno Infantile, Facoltà di Medicina e Chirurgia

Università degli Studi di Brescia

cena@med.unibs.it 
quello di mantenere le diverse professionalità in costante aggiornamento lungo tutto l'iter professionale 2 .

In questa prospettiva indichiamo la succitata indagine esplorativa (capitolo 11) nell'ottica di una ricerca-intervento: ricerca per la formazione delle figure professionali socio-sanitarie addette ai percorsi nascita; intervento per fornire un servizio di prevenzione al territorio, in sinergia con le altre agenzie sanitarie ed educative (ASL, Consultori, Ospedale, Scuola) che operano con la finalità di perseguire la tutela della salute mentale e la promozione del benessere psicofisico della donna, del bambino, della coppia e della famiglia.

\subsection{Genitori: progettualità generativa, genitoriale, transgenerazionale}

La Psicologia Clinica Perinatale ha come suo oggetto unitario lo sviluppo della progettualità generativa e genitoriale di una triade madre-bambino-padre e il suo evolversi dall'epoca prenatale sino ai primi anni di vita di un bimbo nell'insieme relazionale che li lega, collocato nel collettivo costituito dall'organizzazione dei servizi assistenziali istituiti per proteggere e aiutare il processo di nascita, nonché nel più vasto contesto culturale e sociale. L'ottica in cui si muove è quella di un approccio multidisciplinare in cui la complessità del fenomeno dello sviluppo del feto, del neonato e del bambino viene considerato non tanto nei suoi aspetti psicobiologici quanto relazionali. L'oggetto della Psicologia Clinica Perinatale non è pertanto il bimbo nella sua individualità, ma come questa si sviluppi, fisicamente e psichicamente in funzione della relazione che si costruisce con i suoi genitori. Alla base vi è il con-

${ }^{1}$ Nei percorsi di formazione psico-pedagogica e clinica dei Corsi di Laurea in Ostetricia dell'Università di Brescia operiamo sia con strumenti di osservazione clinica e pedagogica dell'unità madre-bambino e della coppia, sia con strumenti-questionari, interviste, colloqui clinici- per la rilevazione dei fattori di rischio e di protezione del benessere psicofisico perinatale della madre, del bambino e del padre . Con la coordinatrice del Corso di Laurea in Ostetricia, Prof.ssa Miriam Guana, ostetriche, puericultrici degli Spedali Civili di Brescia, ginecologi-ricercatori del Dipartimento Materno Infantile dell'Università di Brescia e assistenti sanitarie dell'Asl di Brescia, da diversi anni stiamo collaborando in questa direzione con progetti di ricerca e di formazione per un'integrazione degli aspetti psicofisici dell'esperienza perinatale genitoriale, nella nascita a termine (II Divisione di Ginecologia e Ostetricia-Direttore Prof. Pecorelli, Dott.ssa Gambino Angela; I Divisione di Ginecologia e Ostetricia- Dott. LoJacono Andrea) e pretermine (Reparto Terapia Intensiva Neonatale: Prof. Chirico e Dott.ssa Angeli; Reparto Nido: Dott.ssa Gasparoni), che ringraziamo per la disponibilità e l'impegno nella collaborazione.

2 Da diversi anni ci stiamo impegnando in questo ambito attraverso la proposta di Congressi Nazionali e Internazionali, Corsi di Perfezionamento e di Aggiornamento che vedono coinvolte professionalità specialistiche su aree tematiche relative alla perinatalità. Il presente testo è un contributo degli atti del secondo Corso di Aggiornamento (il primo nel 2008 e il secondo nel 2011) rivolto alle professionalità socio-sanitarie del territorio bresciano, italiano e internazionale tenuto da Patricia Crittenden del Family Institute, fondatrice dell'International Association for the Study of Attachment (IASA) e da Andrea Landini, Neuropsichiatra Infantile-IASA, con cui abbiamo da diversi anni una collaborazione per la formazione e la ricerca. 
cetto di continuità tra la vita intrauterina ed extrauterina, comprovata dalla possibilità di osservare in vivo la complessità delle attività e delle prerogative manifestate dal nascituro fin dai primi tempi della gestazione, e la conoscenza di quanto questa sia dipendente dalla relazione che si stabilisce coi genitori.

Freud (1926) sottolineava che tra la vita intrauterina e la prima infanzia vi è molta più continuità di quel che non lasci credere l'impressionante cesura dell'atto della nascita. L'attenzione della Psicologia Clinica Perinatale alle vicende della gravidanza, gestazione, parto, nascita, allattamento, neonatalità, crescita e sviluppo del bambino, copre l'arco di vita che va dall'epoca prenatale fino ai primi 2 anni di vita del bimbo, restando tuttavia interessante poter mantenere l'osservazione clinica almeno fino ai 36 mesi di vita. Il bambino non è considerato individualmente, ma nell'ambito del contesto interattivo diadico e triadico, secondo quanto intuito da Winnicott che "non esiste il bambino come entità a sé" (1965). Questo per due ordini di ragioni, che rimandano entrambe alla modulazione relazionale: la prima riguarda lo studio della regolazione (psicosomatica) del concepimento e di tutte le successive vicende, nella normalità di una gestazione e di un parto detti fisiologici, con una loro notevole gamma di ottimalità-difficoltà, nonché nella patologia, mentre la seconda rimanda allo sviluppo mentale primario (feto e neonato) quale condizione dello sviluppo dell'individuo in formazione, in cui la massima attenzione viene rivolta alla relazione, madre-feto/neonato-padre. Il periodo perinatale di un bambino è indissolubilmente legato, forse determinato, dalla maturazione psichica che avviene, o dovrebbe avvenire, nella struttura mentale dei suoi genitori: si può dunque considerare, e studiare, una perinatalità psichica come caratteristica fondamentale dei genitori condizionante, nel suo evolversi, il futuro del bimbo e dell'intera sua famiglia.

La Psicologia Clinica Perinatale si occupa dunque innanzitutto della perinatalità psichica dei genitori: il periodo della vita della donna e della coppia, contrassegnato dalla perinatalità fisiologica (concepimento, gravidanza, parto, puerperio), caratterizzato da una sottostante processualità psichica che si struttura progressivamente nella mente di ogni membro della coppia, durante il periodo della gestazione, ma anche già da prima, a partire dalla progettualità generativa che aveva motivato consapevolmente o inconsapevolmente il desiderio di un figlio.

I processi psichici sottesi alla filiazione sono costituiti dai vissuti intrapsichici dei futuri genitori, ma sono anche collegati al contesto sociale e culturale. La perinatalità psichica viene pertanto a collocarsi in una prospettiva ampia, che implica aspetti intrapsichici, interpersonali, relazionali, sociali e transgenerazionali. Essa fa parte di quel complesso quadro denominato "transizione alla genitorialità". I recenti mutamenti sociali e culturali, che hanno consentito la diffusione di nuove strutture familiari e modalità diversificate di assunzione del ruolo genitoriale, hanno proposto percorsi della filiazione sempre più articolati (procreazione medicalmente assistita, prematurità, differenti condizioni di accudimento ecc.), cosicché gli aspetti connessi alla perinatalità psichica sono andati implicando processi sempre più complessi.

Collocati entro il contesto più ampio della genitorialità psichica, i processi psichici della perinatalità implicano riconoscere un momento critico nel ciclo di vita dell'individuo, collegato a un intenso lavoro di ristrutturazione emotiva ed affettiva. La "nascita" psicologica di un genitore, ovvero la genitorialità psichica, fa parte di un 
percorso evolutivo articolato e complesso, attraverso un divenire di processi tra loro interrelati, a partire dal proprio senso di identità individuale e poi di coppia, dalla gestione della propria sessualità, dalla capacità o impedimento di procreare, dal desiderio di generare e di maternità/paternità, e dalla possibilità di allevare un figlio che si possono ritrovare sotto molteplici dimensioni nella storia particolare e unica di ogni individuo (Cena, Imbasciati, 2007; Imbasciati, Cena, 2011).

L'appartenenza alla specie umana implica potenzialità generative psichiche, con cui è possibile trasmettere transgenerazionalmente caratteristiche della propria discendenza parentale per via psichica e non semplicemente per trasmissione genetica. Attraverso la propria sessualità ogni individuo ha potenzialmente la possibilità di generare e diventare genitore: anche questo però non passa semplicemente per le vie biologiche, ma nella sessualità intesa come struttura neuropsichica emozionale entro la quale viene ad essere bene o male costruita la potenzialità psichica generativa e genitoriale (Imbasciati, 2011a). La generatività intesa in senso psichico rappresenta una condizione creativa della dimensione psichica sessuale e la genitorialità ne è a sua volta la prosecuzione, nella linea della filiazione psichica, mediante il prendersi "cura" del prodotto del concepimento. Essa è necessaria come la stessa generatività biologica, per la continuazione e la protezione del nuovo essere: nella specie umana, nella situazione di infanzia prolungata e dipendente, il nuovo nato ha la possibilità di strutturare la propria mente nelle relazioni con la mente dei genitori, per potersi poi sviluppare e crescere, e poi ancora nuovamente riprodursi in senso fisico, ma anche e soprattutto in senso psichico, per una continuazione della specie "sapiens".

Alcuni autori parlano di una dinamica esplicita del generazionale che rimanda a una trasmissione implicita tra le generazioni di quella che non è solo un'eredità biologica ma anche psichica (Golse, 1995). Lebovici $(1994 ; 1989)$ fa riferimento a un "mandato transgenerazionale" che costituisce il patrimonio psichico, tramandato come preziosa eredità tra le generazioni, ma che potrà anche costituire un fardello pericoloso e pesante. Le interazioni fantasmatiche tra le generazioni (Kraisler, Cramer, 1981; Lebovici, 1994) sono il veicolo privilegiato di questa trasmissione psichica transgenerazionale, con cui si intesse lo sviluppo intrapsichico individuale.

Alla base di ogni azione umana è sottesa, consapevole o no, una progettualità che guida le scelte e le azioni stesse: gli obiettivi o comunque i risultati potranno essere razionali o irrazionali, espliciti o impliciti, consapevoli o no, sani o patologici. Possiamo pertanto fare riferimento a una "progettualità" generativa e genitoriale per descrivere i processi psichici interrelati e sottesi allo sviluppo delle dimensioni che costituiscono la filiazione umana: il riferimento al concetto di progettualità è rappresentativo della dinamicità dei processi evolutivi implicati. Generatività e genitorialità si presentano come costrutti teorici articolati, imbricanti strutture e funzioni psichiche connesse con molteplici variabili. Il progetto gestazionale e genitoriale (Capello, Vacchino, 1985) della donna e della coppia comporta che attraverso la gestazione avvenga un passaggio dalla "coppia coniugale" alla "coppia genitoriale". Il progetto gestazionale contiene il progetto generativo (fare un figlio) e il progetto genitoriale (diventare madre e padre), che dovrebbe comportare lo sviluppo di un'identità nuova, quella di genitore. Negli studi che da diversi anni stiamo conducendo in quest'area di ricerca (Imbasciati, Dabrassi, Cena, 2007; 2011), abbiamo cercato di 
individuare possibili indicatori di generatività e genitorialità.

La progettualità generativa individuale che appartiene alla storia di ognuno potrà a un certo momento del percorso di vita venire condivisa o meno dalla coppia, o potrà anche nascere come progettualità della coppia stessa, sintetizzando e assumendo i codici individuali. La progettualità generativa consapevole implica comunque processi psichici inconsapevoli, sottesi ai comportamenti relativi alla propria sessualità, che potrà essere modulata nei suoi stessi aspetti psichici, nonché essere gestita con modalità più o meno orientate alla procreatività.

La progettualità genitoriale comporta d'altra parte l'attivazione dei processi psichici strutturati nella mente del singolo, che permettono, bene o male, le funzioni di accudimento, fisico e psichico, del bimbo generato. La genitorialità psichica rimanda alle rappresentazioni di affetti e di comportamenti rivolti al proprio bambino, nato o in gestazione, che può non essere ancora rappresentata, ma essere presente solo nella propria mente (Stoleru, Morales-Huet, 1989). Il processo del diventare genitori comporta una complessa evoluzione psichica che inizia nell'infanzia, l'attraversa e segue nello sviluppo evolutivo dell'individuo che potrà diventare genitore, in complementarietà allo sviluppo dei suoi legami generazionali, sociali, ambientali (Moro et al., 1989). In termini psicodinamici la genitorialità implica l'attivazione di una relazione oggettuale con un altro da sé, bambino reale o immaginario (Stoleru, 1998), e comprende la sinergia delle due evoluzioni psichiche della maternità e della paternità, che si incontrano nello spazio condiviso della coniugalità.

In genere i due processi di attivazione della generatività e della genitorialità sono interconnessi: la generatività può evolvere verso la genitorialità, o viceversa un progetto di genitorialità può attivare la generatività dell'individuo e della coppia: ciò potrebbe spiegare l'interscambiabilità dell'uso dei due termini, i cui concetti sono comunque da tenere ben distinti. Può accadere infatti che, per complesse vicissitudini interiori, intrapsichiche, interpersonali o anche sociali, la generatività sia debole o non sia sostenuta, o non evolva verso la genitorialità: il ruolo genitoriale può venire in questo caso assunto da qualche altro caregiver, entro o fuori la famiglia, oppure nei casi più gravi il bimbo può venire abbandonato, maltrattato, abusato o ucciso.

Può anche accadere però il contrario: il progetto di generatività può fallire per impossibilità procreativa, fisiologica o psicologica di uno o di entrambi i membri della coppia. La genitorialità può implicare allora aspetti particolari, indipendenti dalle possibilità generative biologiche. Basti pensare alle coppie infertili in cui il progetto di genitorialità può venire realizzato solo attraverso l'adozione di un bambino o l'assunzione di un affido.

In letteratura si possono ritrovare i due termini generatività e genitorialità utilizzati in modo interscambiabile. Con le due denominazioni si intendono però diversi e specifici processi psichici, che necessitano di una distinta puntualizzazione. Per generatività si intende una struttura psichica, che dispone a una qualche trasmissione del proprio essere: può condurre alla generatività fisica, così come ad altre "creazioni"; rimanda al superamento del narcisismo individuale a favore di un qualcosa, cui l'individuo può anche sacrificarsi. In questi termini è stata descritta da Erikson (1950) come ulteriore sviluppo di una "genitalità", intesa come oblatività e dedizione reciproca. Nei suoi aspetti più concreti generatività rimanda a gravidanza, e da 
qui, superando il narcisismo della propria capacità di generare fisicamente, rimanda a un voler portare avanti una gestazione, al voler "fare un figlio": desiderio presente in entrambi i sessi, che ritroviamo celebrato nel corso dei tempi nei miti e nelle cosmogonie (Imbasciati, Cena, 1987, 1988). La genitorialità implica invece un ulteriore sviluppo della generatività, che non sempre avviene e che comunque assume aspetti più individualizzati, che sfociano non tanto nel desiderio di un figlio, quanto in una serie di rappresentazioni che concernono l'allevamento di un figlio e il desiderio di aiutarlo a diventare adulto a sua volta.

In letteratura il desiderio femminile di procreazione si ritrova principalmente declinato attraverso un duplice aspetto: come desiderio di gravidanza e desiderio di maternità (Pines, 1972, 1982; Baruffi, 1979), aspetti diversi che rimandano a quelli della generatività e della genitorialità. Shaffer (1980) e Badinter (1981) rilevano come non si possa parlare di un comportamento istintivo che conduca la donna verso la maternità, ma di un insieme di capacità e sentimenti basati su una disponibilità interiore della madre, che si manifestano in particolari condizioni psicologiche e sociali (Vegetti Finzi, 1997).

Il desiderio di generare può essere consapevole e condurre a un'azione volontaria: il concepimento viene cercato, programmato, desiderato e atteso dall'individuo e dalla coppia, ma può essere anche poco o nulla consapevole, e dare origine a tutta una serie di azioni non palesi e talora occulte per l'individuo.

Il desiderio di generare viene spesso fatto risalire all'istinto: negli animali infatti il comportamento riproduttivo è regolato dai ritmi fisiologici dell'estro che segnano la possibilità ciclica di generare nella femmina. La riproduzione procede sulla base di una regolazione istintuale uguale in tutti gli individui della stessa specie. È questo che definisce l'istinto: tale comportamento istintivo, che regna nel mondo animale, della femmina che ricerca l'accoppiamento quando è in una situazione di estro e dell'incoercibilità del comportamento coitale del maschio quando percepisce una femmina in estro, è scomparso per il genere umano. Del resto il concetto di istinto anche nei suoi moventi più generali, non è applicabile all'uomo (Imbasciati, Ghilardi, 1990; Ghilardi, Imbasciati, 1989). Ciò nonostante nella cultura attuale corrente si fa riferimento a un implicito "istinto sessuale materno" (Shaffer, 1980), nonché in genere all'istinto: è questa attribuzione errata, corroborata dal confondere gli automatismi appresi inconsapevolmente come procedenti da un supposto "istinto" (Imbasciati, Buizza, 2011).

L'idea di un istinto dietro le vicissitudini dello sviluppo individuale umano è implicita nella prima psicoanalisi, in cui un punto di vista biologico rappresentava un principio fondamentale, che lo studio della riproduzione dava modo di estendere. $\mathrm{Si}$ è così supposto un substrato biologico, istintuale, come giustificazione fisiologica della motivazione riproduttiva. Nello sviluppo della teoria psicoanalitica la questione dell'istinto è stata oggetto di una lunga controversia. Freud faceva riferimento all' "istinto che vuol generare" (Freud, 1915) senza peraltro che fosse precisata la definizione dell'istinto. Note sono a oggi le critiche e le contestazioni a tale modalità di intendere l'istinto, rimaste tuttavia retaggio di un certo senso comune, così come note sono le critiche alla generale concezione biologistica di Freud (Imbasciati, $2005,2007,2010,2011 b)$. Il concetto di istinto è applicabile solo per gli animali in- 
feriori: nei mammiferi subentrano gli apprendimenti (Imbasciati, Ghilardi, 1990; Ghilardi, Imbasciati, 1989). Nell'uomo non si può parlare di istinto: ciò che sembra istintivo è in realtà automatismo appreso in epoche precoci.

Man mano che la psicoanalisi si è evoluta, dalle concezioni endogeniste, e quindi istintiviste, a teorizzazioni relazionali, si è affermata sempre più la nozione che quanto prima era stato ritenuto spinta endogena, ancorabile quindi al biologico nell'antico concetto di istinto, era invece dovuto a uno sviluppo psichico interpersonale, ovvero ad apprendimenti relazionali precocissimi, costituenti memoria implicita. Questa, come tale, muove l'essere umano senza che egli ne possa avere coscienza alcuna (Imbasciati, 2008). Di qui l'errata concezione istintuale: ciò di cui non si ha coscienza, appare automatico e ne facilita l'attribuzione al biologico.

L'idea di generatività/genitorialità inizia molto presto nell'immaginario e nella storia dello sviluppo intrapsichico e dei rapporti interpersonali di ognuno dei due membri della coppia. La generatività è molto più di un evento biologico: è correlata ai vissuti esperiti con le proprie figure genitoriali. Fanno parte di questo bagaglio tutte le fantasie di accoppiamento e di vita di coppia. Successivamente, quando nella vita della coppia sopraggiunge un periodo in cui il "desiderio di fare" dei bambini comincia ad affacciarsi alla coscienza, nella donna e nel suo partner si animano le fantasie inconsce e remote che appartengono alla storia individuale di ognuno di loro (Palacio-Espasa, 1991). Il desiderio di procreazione appartiene del resto già alla vita psichica dei bambini, e questo ancora prima che essi abbiano raggiunto la maturazione fisiologica riproduttiva. La letteratura psicoanalitica evidenzia che la presenza o meno del desiderio di generare sia legato a fasi cruciali dello sviluppo psicosessuale infantile, nelle quali si realizzano processi mentali profondi relativi all'acquisizione della propria identità, in particolare quella femminile.

Nel desiderio di generare sono sottese dinamiche della propria infanzia e dei primitivi rapporti con la madre. Per la donna si tratta di quelle fantasie inconsapevoli che appartengono alla sua infanzia e adolescenza, alle sue identificazioni con le figure amorevoli, che contribuiranno poi a dare un nome e una caratterizzazione fisica al futuro bambino (Ammaniti, 1992).

La genitorialità richiama i processi interiori del "prendersi cura di”, del curare e dell'accudimento del bimbo: secondo Stern (Stern, 1995) nella cura del figlio la coppia farà riferimento alle esperienze di accudimento che aveva a sua volta ottenuto dai propri genitori, riattualizzando antiche modalità di rapporto. Una prova evidente la vediamo nella trasmissione degli stili di attaccamento.

Il desiderio di genitorialità si svilupperebbe anche a prescindere dalla sua evoluzione verso una generatività biologica concreta. Già secondo Freud (1931) il bimbo manifesterebbe una prima identificazione con la madre che comporterebbe un primo comportamento materno per i bambini di entrambi i sessi: da questa fonte di identificazione si svilupperebbero poi i sentimenti genitoriali. Comunque la genitorialità si sviluppa come un processo psichico che accompagna l'esistenza dell'individuo, le cui motivazioni hanno origine nella relazione che $\mathrm{i}$ bambini hanno sperimentato con i propri genitori. Un legame positivo e un'identificazione positiva con i propri genitori possono consentire di generare e di diventare buoni genitori, portatori di un rapporto originario con il proprio figlio (Brazelton, Cramer, 1991). 
La genitorialità rimanda inoltre al "ruolo" di genitore, all'interno del contesto intergenerazionale della famiglia (Scabini, 1985). Con la nascita del bimbo il progetto di generatività può concretizzarsi e consentire una piena realizzazione anche del progetto di genitorialità, attraverso quella serie di modificazioni relazionali, familiari, prima solo potenzialmente inferite, che possono essere sperimentate fattivamente quando nel ciclo di vita dell'individuo e della coppia è venuto il tempo che questo accada.

I vari percorsi della filiazione sono costituiti dalla riedizione e dal trasferimento di contenuti e di modalità di funzionamento psichico, che si demandano e si riattivano per via transgenerazionale (Zurlo, 2009): ciò che ogni membro della coppia genitoriale ha ricevuto dai propri genitori verrà ritrasmesso a sua volta ai propri figli, con tutto il lavoro emozionale che tale processo psichico comporta nel passaggio da una generazione a un'altra. Attraverso i legami della filiazione vengono trasmessi aspetti culturali condivisi dal gruppo di appartenenza su cui si strutturano i processi individuali di identità: in ogni cultura è rilevabile un codice corporeo implicito con cui vengono regolate le modalità di accudimento dei figli, di regolazione emotiva, di comunicazione (Zurlo, 2009): queste dimensioni implicite comuni e condivise regolano ciò che viene trasferito mediante i legami della filiazione, e costituiscono una base comune condivisa dal proprio gruppo sociale da cui prendono origine i processi di individuazione e di costruzione delle identità individuali. A partire dalla famiglia, primo nucleo sociale, la cultura è tramandata nell'ambito delle relazioni primarie, delle interazioni con la madre e i genitori in primis.

Le funzioni psichiche individuali sono pertanto influenzate dalle dimensioni implicite comuni e condivise dal proprio gruppo sociale. Si prospetta un circuito in cui le modalità culturali, caratterizzate da regole, valori, riti, norme, credenze influenzano l'individualità che, a sua volta, li rielabora e li rappresenta attraverso i propri vissuti personali (Zurlo, 2009).

\subsection{Viaggi di "andata e ritorno" nella perinatalità psichica dei genitori: quando nascono i genitori?}

La vita psichica evolve entro contesti intersoggettivi, familiari, transgenerazionali e sociali e lo sviluppo psichico individuale si struttura entro una rete di "appoggi " e "sostegni multipli" (Kaes, 1982). Tale modalità di supporto è stata descritta con modalità diverse da autori secondo più vertici teorici di riferimento: in psicoanalisi viene descritta come capacità di contenimento (Winnicott, 1965) o di rêverie (Bion, 1962); secondo la psicologia dello sviluppo viene indicata come supporto, impalcatura o scaffolding (Bruner et al., 1976), oppure come spazio in cui l'altro, il sociale, può partecipare ai processi elaborativi individuali per supportarne i processi trasformativi, all'interno di quella zona definita "sviluppo prossimale" di un individuo (Vygotskij, 1990). Le diverse teorie concordano nel ritenere, seppure con vertici diversi di comprensione e di intenti pedagogici o clinici, che la funzione di questi sostegni non sia solo supportiva, bensì trasformativa in quanto essi sono operanti entro l'intersoggettività.

In particolare, in relazione ai processi relativi alla perinatalità psichica genitoria- 
le, ci interessa rilevare il concetto di "sostegni multipli" di Kaes, in cui si fa riferimento a tre ordini di esperienze che strutturano l'individuo: la prima è riferita all'esperienza soggettiva corporea, che alimenta la vita psichica; un secondo ordine di esperienze strutturanti sono quelle relazionali che strutturano le modalità di funzionamento psichico materno e paterno; un terzo ordine di esperienze si riferisce al più vasto sostegno che proviene dal sociale. Questa concettualizzazione di Kaes può essere funzionale per riflettere sui processi che caratterizzano la filiazione (Zurlo, 2009): in primo luogo le funzioni strutturanti l'identità dell'individuo si costruiscono a partire dalle esperienze corporee, comprese quelle relative alla propria sessualità maschile e femminile; l'altro ordine di esperienze è riferito a quelle relazionali, in particolare all'implicito riemergere delle esperienze di accudimento fisico e psicologico ricevute dalla propria madre e dai propri genitori: un terzo ordine di esperienze è riferito al coinvolgimento del sociale. Quest'ultimo comporta che attraverso il riconoscimento della collettività alle necessità del singolo, della coppia e della famiglia, vengano forniti sostegni attraverso le istituzioni e le organizzazioni sociali che intervengono a supporto delle funzioni generative e genitoriali, mediante figure professionali come medici, neonatologi, pediatri, ma anche di altri specialisti, come neuropsichiatri infantili, psicologi clinici della perinatalità, ostetriche, infermieri, fisioterapisti, tecnici della riabilitazione funzionale, operatori sanitari e tutto il personale specializzato che si occupa della perinatalità nei reparti ospedalieri e nei servizi consultoriali e sociali.

La psiche individuale si sviluppa su una molteplicità di sostegni: il sostegno delle formazioni culturali sociali (istituzioni, associazioni sociali) influenza lo psichismo integrandosi con altri sostegni intrapsichici. L'elaborazione delle esperienze personali è regolata dalle relazioni interpersonali consapevoli e inconsapevoli e i processi di identità individuale si strutturano a partire dai processi di identificazione con l'altro e dalle rappresentazioni trasmesse per via transgenerazionale, che costituiscono la base della individualità del soggetto e consentono una continuità di trasferimento dei contenuti, dei significati, degli ideali, delle credenze e dei processi psichici del gruppo familiare e sociale. La funzione del figlio e la sua collocazione entro il contesto transgenerazionale viene definita dal gruppo sociale a cui esso appartiene, che in tal modo si assicura la continuità di quella che si potrebbe definire la propria eredità psichica, che si tramanda e rimane attiva nella psiche individuale.

La perinatalità psichica è un periodo in cui avviene nella coppia la rielaborazione delle problematiche relative alla propria identità e si delinea come un ritorno alla genesi del proprio sviluppo mentale con le vicissitudini e i conflitti che ne avevano accompagnato l'iter evolutivo. In particolare nella donna si intensifica la capacità di entrare in contatto col proprio mondo interiore e i propri vissuti relativi al passato attraverso quella che la Bydlowski definisce come un'esperienza di trasparenza psichica (1991). L'autrice rileva come nel periodo perinatale questa maggiore "trasparenza psichica" della donna, che le consente una maggiore sensibilità e attenzione ai propri processi interiori e ai cambiamenti, la mette però anche in una condizione di maggiore vulnerabilità e di rischio (1989), perché può favorire il riemergere di antiche ferite affettive (1997) di fronte alle difficoltà e alle problematiche che la donna deve affrontare in gravidanza, come le trasformazioni somato-psichiche, le interazioni reali e fantasmatiche feto-materne, le immagini ecografiche, il dolore del parto, precedenti trau- 
mi o eventuali complicanze ostetriche (Bydlowski, Raul-Duval, 1978).

La gravidanza è l'esperienza psicosomatica per eccellenza (Imbasciati, Cena, 2007a) in cui vi è una circolarità fra dimensioni fisiche e psichiche, dove un evento psichico dai mutevoli gradi di consapevolezza, il desiderio di avere un figlio, comporta la disponibilità da parte della donna di rimanere gravida, permette la gestazione che a sua volta promuove o incentiva processi mentali, in particolare di rielaborazione delle proprie esperienze passate, di progetti e aspettative future. Questi processi hanno una ricaduta somatica nell'andamento della gravidanza e del parto: la Deutsch, (1945), la Bibring, (1959), la Benedek (1958), la Soifer (1985), la Pines (1972) hanno esplorato le varie fasi evolutive della gravidanza e messo in evidenza aspetti di crisi e sviluppo. Altri autori hanno esplorato invece aspetti interiori delle rappresentazioni presenti nella mente delle madri e dei padri (Ammaniti et al., 1995) o messo in evidenza come la donna, il suo partner e il bimbo costituiscano una particolare "popolazione clinica" e come tale vada esaminata (Stern, 1985); mentre molti altri autori ancora ci hanno consentito di avere acceso agli aspetti peculiari della perinatalità psichica della coppia e alla sua comunicazione con il feto (Capello, Vacchino, 1985; Imbasciati, 1990; Manfredi, Imbasciati, 2004; Cena, 2005a,b; Imbasciati, Cena, 2007b).

Gli autori concordano come il progetto di filiazione della coppia sia caratterizzato dal riemergere nella psiche di rappresentazioni riferite sia all'essere genitore, con una identificazione alla genitorialità dei propri genitori e un coinvolgimento affettivo ed emotivo nei loro confronti, sia contemporaneamente con una identificazione al bimbo desiderato o generato e al riemergere di rappresentazioni relative a se stessi come bambini.

Altri autori (Darchis, 2009) fanno riferimento a questi processi rivolti alla costruzione della genitorialità, presenti nei normali percorsi della filiazione, con la metafora di un viaggio, per descrivere i movimenti dinamici che li caratterizzano: questa metafora si presta molto bene a illustrare il processo di esplorazione e rielaborazione emotivo-affettiva entro cui si avventura la coppia, la donna e il suo partner, quando si apprestano a diventare genitori. Indispensabile è il riferimento alla coppia, perché comunque nei percorsi della generatività un bimbo viene generato da un padre e da una madre ed entrambi sono comunque presenti, almeno psichicamente. La metafora del viaggio ci consente di rappresentare i processi della perinatalità psichica in cui avvengono processi di riorganizzazione psichica dell'identità individuale, maschile e femminile, di coppia e transgenerazionale. Il viaggio metaforico che la coppia genitoriale intraprende implica due diversi percorsi di esplorazione: di andata e ritorno all'interno di se stessi. Questi processi sono caratterizzati da una doppia identificazione. Il viaggio comporta una proiezione verso il futuro e contemporaneamente un ritorno al passato. Sempre tenendo presente il modello dei "sostegni multipli" dello psichismo (Kaes, 1982), nel primo processo identificatorio inteso come orientato verso il futuro, ogni membro della coppia genitoriale si appoggia ai sostegni multipli su cui ha costruito la propria identità personale per farne una base di supporto e sostenere l'identità del bambino: i movimenti sono costituiti da una identificazione della madre con sé stessa bambina, che le consente una identificazione con il bimbo che ha in gestazione; analogamente il padre affonda le radici nel contenitore psichico del suo gruppo familiare per poter rivolgersi al futuro. Se questa rete è 
stata sufficientemente adeguata a sorreggere, attraverso la sue maglie relazionali, la coppia genitoriale, questa potrà avere quella sufficiente sicurezza per rivolgersi al futuro, e costituire a sua volta un nuovo contenitore psichico familiare. Attraverso un avvicinamento e una differenziazione dal contenitore psichico della propria famiglia, i nuovi genitori potranno strutturare un nuovo contenitore psichico per il loro bimbo e collocarsi all'interno come genitori.

Nel secondo movimento identificatorio, nel viaggio metaforico di ritorno al passato, i due membri della coppia genitoriale hanno necessità di ritrovare i propri genitori, quelli reali e quelli delle proprie rappresentazioni interiori, avvicinandosi ad essi per prendere poi da questi le giuste distanze e costruire una propria identità genitoriale. In questo processo di trasformazione identitaria (Darchis, 2009) la riorganizzazione psichica consente l'accesso a nuovi ruoli: di se stessi come genitori, dei propri genitori vissuti come nonni, e del bimbo come figlio. Il genitore nasce contemporaneamente al proprio figlio.

Questo viaggio verso il passato per ritrovare il "terreno psichico" delle proprie origini identitarie comporta però anche dover riaffrontare antiche conflittualità sopite, sofferenze dovute a traumi o lutti irrisolti: il rincontrarsi con la propria infanzia e i propri genitori può allora risultare doloroso, a un punto che il viaggio può risultare addirittura impossibile da iniziare, o non giungere mai a termine, e l'assunzione del nuovo ruolo genitoriale può non venire intrapresa. Possono presentarsi ostacoli psichici inconsapevoli alla generatività, sia attraverso modalità di evitamento che si manifestano con interruzioni volontarie o patologiche della gravidanza e/o infertilità psicogene sia, dopo la nascita del figlio, attraverso difficoltà relazionali e di accudimento del bimbo, con una trasmissione transgenerazionale di traumi irrisolti. Ci possono essere profonde lacerazioni nei percorsi della filiazione che spesso impediscono la transizione alla genitorialità, paralizzandone il processo evolutivo.

Quando per problematiche che possono sopraggiungere nel periodo perinatale il bimbo, ad esempio, viene partorito prematuramente, il viaggio verso la genitorialità intrapreso dai genitori subisce deviazioni inaspettate o viene bruscamente interrotto: una nascita prematura spesso implica una genitorialità prematura. Un buon incontro genitore-bambino è reso possibile da un compiuto viaggio dei futuri genitori nel proprio passato infantile, percorso che serve a rielaborare le identificazioni durante la gravidanza: con una nascita prematura il genitore può non essere ancora sufficientemente pronto a questo incontro. La ricerca della identità genitoriale viene descritta dalla Darchis con la metafora dell'odissea che deve intraprendere un genitore per poter diventare tale: la Darchis individua come complesso di Telemaco (Darchis, 2009) il processo che struttura la psiche del futuro genitore, in cui egli deve riuscire a recuperare le sue radici per legarsi ad esse, al fine di separarsene meglio. Nell'Odissea Telemaco, il figlio di Ulisse, parte all'avventura per conoscere il destino di suo padre e poter a sua volta crescere.

I due movimenti identificatori verso se stesso come bambino, per riconoscere il proprio bambino, e verso i propri genitori per costruire la propria identità di genitore, possono non giungere a buon fine, quando la nascita è pretermine: può verificarsi un trauma o un forte stress, con uno sconvolgimento emotivo, e il viaggio allora può interrompersi o bloccarsi. 
La costruzione del primo legame con il bimbo inizia già prima della nascita, durante la gestazione (Brazelton, Cramer, 1991). Recenti esplorazioni cliniche hanno messo in evidenza come, alla nascita esso progressivamente attraversi tre stadi integrativi: il primo stadio è quello del primo incontro in cui deve avvenire "l'adozione" del proprio bambino da parte dei genitori; il genitore compie un processo integrativo tra la propria identità di padre e madre e quella del figlio. Un secondo processo integrativo in questo primo stadio è quello tra la rappresentazione del proprio bambino immaginario, il bimbo della notte (Vegetti Finzi, 1990) e il bimbo reale. Nella nascita prematura spesso il bimbo reale è ancora un estraneo, può inoltre suscitare terrore nel genitore che non lo riconosce e che può percepirlo come un oggetto persecutore mostruoso (Darchis, 2009). Il genitore pensa che "quello lì" non può essere il suo bambino: il viaggio verso il passato alla ricerca di una identificazione con se stessi bambini non ha funzionato. Il genitore non riesce a ritrovare nel passato se stesso, per poter riconoscere il suo bambino. Così pure non riesce a identificarsi con il proprio genitore, che invece non lo ha generato in modo immaturo. Antichi traumi nell'ambito della propria storia o provenienti per via transgenerazionale possono inoltre venire riattivati da questa nuova esperienza traumatica. Nei casi più dolorosi, può avvenire anche un'esplosione dell'identità del genitore (psicosi puerperali): il legame della filiazione non riesce a instaurarsi.

Un'altra eventualità, che potrebbe verificarsi sempre nel primo stadio, è che il legame di filiazione si possa instaurare secondo modalità fusionali: il genitore non riconosce l'esistenza separata del bambino, le sue parti infantili trovano espressione diretta nel neonato stesso. Attraverso processi di identificazione proiettiva egli cura e protegge parti sofferenti del proprio sé, nel bimbo immaturo. Si occupa del bimbo allora molto rapidamente, ma in modo molto apprensivo ed esagerato, spesso non lasciando spazio all'altro genitore. Solo attraverso un sostegno terapeutico il genitore può venire aiutato a costruire la sua genitorialità secondo quelle che sono le esigenze del bimbo reale, e in modo adeguato intraprendere o concludere la propria odissea.

Quando "l'adozione" del bimbo si è realizzata, il genitore può accedere al secondo stadio e funzionare secondo modalità inerenti il suo nuovo ruolo, attraverso l'accudimento del bimbo. Questo implica che nella madre deve subentrare quell'esperienza interiore descritta da Winnicott come "preoccupazione materna primaria" (1956), che le potrà permettere di essere una "madre sufficientemente buona" (1958): l'unità gestante-feto deve diventare la matrice dello sviluppo psichico del bimbo (Imbasciati, Cena 2007c). La funzione paterna è un indispensabile sostegno in questo processo. Le difficoltà riscontrate più frequentemente possono essere dovute a proiezioni troppo massicce, per cui il genitore non riesce a viversi in un'immagine di sé sufficientemente buona, malgrado le difficoltà occorse: resta così nell'illusione di poter soddisfare in modo onnipotente tutte le esigenze del bimbo e potrà allora adottare modalità maniacali di funzionamento mentale. Nel caso di un bimbo pretermine, che non può essere accudito secondo gli ideali, questi può diventare il nemico che ostacola la realizzazione dei desideri genitoriali onnipotenti.

Un'altra modalità di funzionamento mentale può essere invece di tipo depressivo: il genitore durante il processo di disillusione tra la rappresentazione del bimbo immaginario e quello che col pretermine gli si è presentato reale, viene sopraffatto. 
Sente di non essere stato sufficientemente adeguato e vive se stesso come un cattivo genitore: un genitore che ha generato un bimbo imperfetto. Insicurezze nei propri movimenti identificatori con una perdita delle rappresentazioni genitoriali positive possono condurre a effetti depressivi. La prematurità in questi casi rafforza il sentimento di inefficacia del genitore. Il padre allora può diventare un sostegno molto valido e contribuire alla prevenzione di una depressione materna, oppure colludere con questo sentimento materno di inadeguatezza e intensificarlo. La relazione genitore-bambino sarà a rischio di maltrattamenti o abbandoni.

Un buon funzionamento può invece sopraggiungere quando la madre, superati i sentimenti di inadeguatezza, si adatta al bimbo, abbandonando le sue rappresentazioni del bimbo immaginario e della madre perfetta: l'accudimento e la soddisfazione dei bisogni reali del bimbo rinforzano allora le sue capacità genitoriali, di contenimento e di rêverie (Bion, 1962). In questo caso anche il padre ha svolto una funzione rassicurante e ha sostenuto la funzione di madre sufficientemente buona, consentendo la costruzione di un legame che si potrà consolidare in un legame di attaccamento, via via con la crescita e lo sviluppo del bimbo. Per il bimbo pretermine questa situazione non può che verificarsi in genere con il ritorno a casa dopo l'ospedalizzazione.

Nei percorsi alla filiazione che presentano maggiori complessità, come una nascita prematura, se i processi identificatori genitoriali non si sono adeguatamente conclusi o si sono bloccati, il legame genitore-bambino si può costruire nella confusione delle storie passate e strutturarsi con modalità patologiche di ripetizione di antichi traumi transgenerazionali. La non differenziazione tra genitore e bambino sollecita meccanismi di identificazione proiettiva e non consente un'adeguata sintonizzazione con i reali bisogni del bimbo. Il bimbo è imprigionato dai fantasmi dei genitori: i fantasmi nella camera dei bambini (Fraiberg et al., 1975). La funzione genitoriale allora non è contenitiva dei reali bisogni fisici ed emotivi del bambino, ma sono i genitori ad aver bisogno loro stessi di un contenimento per poter nascere nel loro ruolo di genitori.

Nei casi di maggiori difficoltà, attraverso un supporto terapeutico il procedere delle tappe evolutive della perinatalità psichica potrà avvenire con modalità e ritmi diversi a seconda della dinamica di funzionamento mentale genitoriale. Lo spazio della terapia ha, secondo la Darchis (2009), funzione di matrice contenitiva entro cui possono essere rivissute e rielaborate le emozioni e le identificazioni primitive. Il terapeuta- contenitore consente al genitore uno spazio per riappropriarsi degli eventi traumatici, senza restarne annientato, consentendogli di ripensare ai vissuti e alle esperienze del passato, riconciliandosi con le antiche figure genitoriali, ma prendendo da esse le dovute distanze e differenziandosi: solo allora il viaggio di ritorno potrà concludersi. Il legame genitore-bambino può instaurarsi quando il complesso di Telemaco si risolve e l'odissea dei genitori termina.

\subsection{La relazione primaria genitore-bambino}

In un nostro precedente testo (Cena, Imbasciati, Baldoni, 2010), che consideriamo propedeutico al presente volume, abbiamo cercato di mettere in evidenza come nel 
corso degli anni si sia verificata un'evoluzione degli studi osservazionali e clinici nei bambini, con un progressivo cambiamento da un orientamento rivolto prevalentemente ai processi intrapsichici a una prospettiva relazionale (capitolo 1). Questo ha anche contribuito a un cambiamento nelle diverse modalità di concepire il trattamento, con una modificazione i setting e dei tipi di terapia. Un notevole cambiamento si è verificato nell'ambito della psicoanalisi infantile che, prendendo origine dalla psicoanalisi degli adulti, ha dovuto "adattare" modalità di osservazione e di trattamento ai bambini, trovandosi a ricercare modalità comunicative diverse dal linguaggio parlato che potessero avere effetto mutativo terapeutico con soggetti in un'età in cui il linguaggio non si è ancora sviluppato. Un'attenzione specifica venne attribuita da Melanie Klein (1932) nella terapia dei bambini all'uso del gioco, nella differenziazione di prototipi di giochi nel setting. Winnicott (1965), nel suo sottolineare l'unità relazionale "madre-bambino", aprì la strada al valore essenziale della relazione nel suo significato affettivo profondo e nelle sue modalità dialogiche non verbali quali veicoli del cambiamento terapeutico. Negli anni ' 50 iniziarono a svilupparsi forme di intervento terapeutico sui neonati con le loro madri: celebre e pionieristica fu la metodologia della Infant Observation (Bick, 1964). In Italia autori come Giannakoulas e Giannotti (1985), presso l'Istituto di Neuropsichiatria Infantile dell'Università degli Studi di Roma, hanno contribuito a sviluppare la psicoanalisi infantile, mentre all'interno della Società Psicoanalitica (SPI e IPA) si è andati costituendo un training speciale per gli psicoterapeuti dell'infanzia e dell'adolescenza.

L'osservazione sperimentale dei neonati nel loro sviluppo affettivo e cognitivo, promossa dagli studi dell'Infant Research, ha dato in questi ultimi lustri una svolta considerevole allo studio delle interazioni diadiche primarie genitore-bambino, attraverso l'uso di speciali setting con videoregistrazioni. Bowlby diede inizio allo studio del legame di attaccamento per la strutturazione della mente del bambino e di qui anche per il funzionamento mentale degli adulti.

A questi importanti sviluppi hanno contribuito in questi ultimi decenni le neuroscienze, confermando l'importanza delle osservazioni cliniche sui neonati e degli interventi psicoterapeutici precoci, per gli effetti su ogni successivo sviluppo psichico e psicosomatico dell'individuo. Fin dall'epoca fetale si struttura una relazione, in senso psichico affettivo profondo, tra gestante e bimbo, attraverso la trasmissione di messaggi, prima biochimici e poi sonori, motori, pressori e quindi tattili e visivi: tali messaggi corrispondono ad altrettanti mutamenti nella struttura delle reti neurali: essi pertanto sono i responsabili dell'organizzazione strutturale del cervello. È questo il concetto attuale di maturazione cerebrale, non tanto per codici genetici, quanto per apprendimenti modulati dalla qualità delle relazioni. Un dialogo non verbale, contenente messaggi non traducibili verbalmente eppure incisivi, condiziona il cervello del bambino in maniera ottimale piuttosto che patogena. Un'eventuale patologia, o meglio patologizzazione, della maturazione cerebrale dipende da molteplici fattori, dei quali gran parte sono inerenti alla struttura neuropsichica funzionante nella madre nelle condizioni di accudimento.

La dimostrata incidenza dei caregiver sullo sviluppo neuropsichico del bambino acquista ancor più rilievo nella convergenza di studi catamnestici su come la qualità del primo sviluppo psichico condizioni ogni successivo sviluppo neuromentale del fu- 
turo individuo verso l'ottimalità oppure verso il deficit o le patologie. Si è così sempre più affermato il concetto della relazione primaria quale matrice fondamentale su cui operare terapeuticamente, sia individuando tutte le situazioni a rischio, per poterle curare, sia garantendo adeguate situazioni al bambino, ai suoi caregiver e alla famiglia per promuovere un'ottimalità dello sviluppo. Si è così sviluppata una clinica rivolta, non tanto a curare il singolo, quanto a modificare le relazioni - madre-neonato-bimbo e madre-padre-bimbo - matrici della costruzione della mente e del cervello del futuro individuo. Il paziente non è mai il singolo, ma la relazione, le relazioni.

Una diagnostica psicologica precoce si è affermata nella sua importanza preventiva, mentre stanno tuttora progredendo metodologie psicoterapeutiche per curare le relazioni a rischio: in tale contesto presenteremo alcuni di questi recenti sviluppi.

Il nostro lavoro si colloca in particolare nelle attuali prospettive di ricerca di integrazione tra psicoanalisi e teoria dell'attaccamento (Ammaniti, Stern, 1992; Fonagy, 2001). Fonagy ha evidenziato molto bene come sia possibile un terreno comune condiviso, tra la teoria dell'attaccamento e la psicoanalisi infantile contemporanea: questa base comune è costituita proprio dal riconoscimento dell'importanza dei primi anni di vita nella relazione genitore-bambino. Fonagy propone un'innovativa integrazione tra questi modelli teorici: qui si colloca anche il nostro tentativo di evidenziare come certi nuovi orientamenti evoluzionistici della teoria dell'attaccamento (Crittenden, Landini, 2010) aprano orizzonti per la comprensione e valutazione dei disturbi della relazione genitore-bambino (maltrattamenti, abusi, abbandoni) e delle psicopatologie dello sviluppo, che possano fornire un contributo integrativo importante per altri approcci teorici.

La teoria dell'attaccamento, dalla formulazione iniziale di Bowlby e Ainsworth sino alle recenti riformulazioni da parte di teorici quali Main, George, Kaplan, Solomon, Cassidy, Bretherton, Fonagy, Crittenden, permette di riflettere su una modalità di approccio allo studio delle prime relazioni di cura non solo tra madre-bambino, ma recentemente anche tra padre-bambino (Baldoni, 2005), e sull'importanza dei legami significativi che altri diversi caregiver possono instaurare con il bambino fin dalla nascita.

Gli studiosi dell'attaccamento hanno messo in evidenza il paradigma della "qualità" delle cure genitoriali e della sensibilità e responsività del genitore, considerati fattori protettivi per lo sviluppo del bambino stesso. Il modo con cui un genitore risponde ai bisogni fisici e psichici del bambino, e stabilisce una buona relazione, costituiscono la base del suo sviluppo: in condizioni ottimali si sviluppa una "base sicura". Giuoca in questo il sistema di attaccamento dei genitori nei confronti dei propri genitori: la transgenerazionalità dell'attaccamento ha una funzione fondamentale nella trasmissione della capacità dei genitori, a sua volta appresa dai propri genitori, di offrire cure adeguate al proprio bambino. Gli attuali sviluppi evoluzionistici della teoria dell'attaccamento, in specifico il modello di Patricia Crittenden, hanno messo in evidenza come i diversi pattern di attaccamento, sicuro ma anche insicuro, si siano sviluppati come migliore (o meno peggiore) modo funzionale alla sopravvivenza di quell'individuo in quel determinato contesto. In particolare, il modello Dinamico-Maturativo (Dynamic-Maturational Model, DMM) proposto dalla Critten- 
den offre importanti prospettive di ricerca clinica, poiché prevede nella trasmissione del modello di attaccamento e nella sua valutazione la possibilità di una rielaborazione da parte dell'individuo in momenti diversi del suo ciclo di vita, in relazione a cambiamenti significativi, come la stessa transizione alla genitorialità. Il contributo della Crittenden allo studio dell'attaccamento rimanda principalmente a una ridefinizione della "qualità" dell'attaccamento e all'impiego di un modello probabilistico più che deterministico: focalizzando l'attenzione sulle interconnessioni fra genitorialità e relazioni precoci è possibile attraverso le prospettive offerte da tale modello esaminare il ruolo dei legami fra il bambino e le sue figure di riferimento in una prospettiva di cambiamento. La ricerca minuziosa degli indici che rendono unica ogni relazione che i caregiver stabiliscono con il bambino permette di rilevare in modo accurato quali sono le caratteristiche che contraddistinguono la relazione che il bambino ha con le varie figure di attaccamento, quali il padre, la madre, e altri caregiver che si prendono cura di lui. La metodologia della Crittenden offre interessanti contributi alla valutazione della relazione genitore-bambino, fornendo un valido contributo al tema delle prime relazioni di cura: l'autrice ha strutturato tecniche di indagine prevalentemente di natura qualitativa, con la costruzione e la puntualizzazione di specifici strumenti che consentono una valutazione di indici di rischio nella "relazione". Alcuni sono stati presentati nel precedente volume e altri verranno illustrati, attraverso l'applicazione empirica e clinica, nei capitoli della seconda parte del presente volume.

Il complesso intreccio delle "relazioni" che ogni individuo è in grado di stabilire nel suo ciclo di vita lo porta, quando è adulto, a una relazione duratura di coppia, alla transizione alla genitorialità e a prendersi cura del proprio bambino: è necessario pertanto valutare sia le relazioni che egli aveva avuto da bambino con i propri genitori, sia le relazioni attuali come coppia, attraverso il prendersi cura dell'altro partner; questa capacità in ognuno dei partner dipende infatti sia dalle esperienze attuali come coppia, sia dalle esperienze infantili con i propri genitori. Secondo Bowlby (1988) in una coppia ben funzionante ognuno dei due partner sa rispondere in modo adeguato ai bisogni fisici e psichici dell'altro, incoraggiando la crescita personale e dando assistenza al partner (Feeney, Collins, 2004). Le relazioni adulte sono guidate da stili di attaccamento che hanno origine dalle esperienze infantili dell'individuo. La qualità dell'attaccamento è inoltre associata al riconoscimento della sofferenza propria e dell'altro, all'individuazione delle condizioni di stress, e attiva modalità di sostegno-accoglimento, sia all'interno della coppia, sia verso il proprio bimbo. La qualità dell'attaccamento sviluppata dai due genitori ha dunque una notevole importanza nella gestione delle situazioni di conflitto e di stress della coppia.

Nella transizione alla genitorialità, i cambiamenti che vengono richiesti alla coppia sono notevoli ed importante è, nella qualità del sistema di attaccamento, la capacità di accudimento e le espressioni della sessualità (Crittenden, 2008), che possono costituire fattori di rischio o di protezione per il bimbo in quanto si riflettono nel funzionamento della coppia stessa. Nel periodo perinatale il genitore deve far fronte a un processo di transizione che comporta spesso situazioni di stress, facendo affidamento non solo sulle proprie risorse, ma anche sulla forza del legame di coppia: le strategie di coping utilizzate sono in stretta relazione con i pattern di attaccamen- 
to. Recenti ricerche hanno messo in evidenza (McHale, 2010) come i genitori risponderanno allo stress a seconda della qualità dell'attaccamento nella coppia: a seconda dei vari pattern potranno più o meno far fronte alle nuove esigenze poste loro dalla genitorialità.

Quanto più precoci sono gli interventi a favore della relazione genitore-bambino, soprattutto nel periodo perinatale, tanto più sarà possibile attivare cambiamenti su aspetti che non si sono ancora consolidati, nella modalità di relazione e di accudimento del bimbo: un valido aiuto fornito ai genitori nei mesi critici prima e dopo la nascita del figlio e durante i suoi primi anni di vita può fare molto per aiutarli a sviluppare quella affettuosa e comprensiva relazione col bambino, necessaria per il suo sviluppo fisico ed emotivo. "I primi mesi e anni di vita del bambino sono un periodo critico anche per la formazione di una madre e di un padre; un aiuto, se competente e fornito al momento giusto, può servire a molto e i vantaggi di un trattamento su un bambino molto piccolo ci sono a questo punto ben noti; ora auspichiamo che anche i genitori ricevano un aiuto appena "nati"' (Bowlby, 1979, pag. 21).

\subsection{La cogenitorialità}

Oltre agli aspetti intrapsichici, rappresentazionali e relazionali con cui i clinici affrontano le vicissitudini interiori e interpersonali dei genitori con il loro bimbo, è necessario considerare la dimensione relazionale della coppia genitoriale stessa, quella che viene indicata come cogenitorialità. Un bimbo è il prodotto di due individui, e di più generazioni; il contesto relazionale e poi, in una più ampia prospettiva, quello culturale e sociale (Scabini, 1995), sono i panorami entro cui il bimbo si svilupperà e che richiedono un'attenta analisi a chi si prende cura dei bambini e dei loro genitori.

Le relazioni intrafamiliari con i genitori, i nonni e altre figure significative di riferimento affettivo possono essere una risorsa o un ostacolo allo sviluppo del bimbo, così come le modalità relazionali dei genitori tra di loro possono influenzare le loro competenze genitoriali e avere una ricaduta negativa sul bimbo stesso. Gli studi condotti sulla cogenitorialità sono numerosi, spesso parziali attestando in tal modo sia la complessità del tema, sia l'interesse degli studiosi all'argomento. Nelle recenti ricerche sperimentali si fa riferimento al recente costrutto della "cogenitorialità" (McHale, 2010) per intendere la relazione tra i due genitori che si coordinano insieme nella condivisione del processo di accudimento fisico e psichico del proprio bambino.

Le prime ricerche osservazionali sulle dinamiche cogenitoriali precoci provengono da studiosi di terapia familiare che hanno preso in considerazione i conflitti cogenitoriali (Minuchin, 1974), e hanno iniziato a mettere in evidenza come le dinamiche cogenitoriali siano collegate, ma anche distinte, con altre dinamiche di altri sistemi relazionali nella famiglia.

Il costrutto della cogenitorialità può essere affrontato da molteplici punti di vista e secondo vertici di riferimento teorico diverso. In letteratura un recente contributo multidisciplinare longitudinale osservazionale delle interazioni tra il bambino e gli adul- 
ti che si prendono cura di lui, a partire dalla nascita, proviene dagli studi di McHale (2010), che ha analizzato la complessità della cogenitorialità connessa con lo sviluppo del bambino prendendo in considerazione il processo evolutivo che negli individui porta allo sviluppo della cogenitorialità, attraverso traiettorie evolutive diverse su cui esercitano la loro influenza molteplici fattori di rischio, protezione e mediazione. L'autore mette in evidenza il modo in cui agiscono le connessioni tra la qualità della relazione di coppia, la qualità della loro genitorialità e le conseguenze sullo sviluppo del bambino nei suoi primi tre anni di età, e rileva come i micromovimenti che confluiscono in conflitti e disaccordi tra i partner influenzino la relazione che ciascuno di loro ha con il figlio. La qualità della cogenitorialità influisce sullo sviluppo ottimale del bambino: obiettivo di studio è fornire numerosi esempi per aiutare i genitori a cambiare il proprio modo di pensare alla relazione di coppia mentre sono orientati ad accudire il proprio bambino. Questa nuova prospettiva può aprire interessanti orizzonti ai clinici che si prendono cura dei bambini e dei loro genitori durante il periodo perinatale, fornendo linee guida per il tipo di interventi da adottare.

In letteratura si fa riferimento alla "genitorialità condivisa" per indicare come i partner si spartiscono i ruoli - chi fa che cosa - cioè la divisione dei compiti nella crescita del bambino, intendendo le singole azioni dell'accudimento: nutrimento, cambiare il pannolino, farlo addormentare, calmarlo quando piange, e soprattutto interagire e giocare con lui. Questa genitorialità "condivisa" viene distinta da ciò che viene identificato come "cogenitorialità" termine con cui si fa riferimento all'accordo reciproco dei due partner, alla coordinazione e al reciproco sostegno psicologico nell'affrontare le responsabilità genitoriali: il termine "reciproca responsabilità" è parola chiave per identificare questa funzione fondamentale della cogenitorialità.

McHale ha considerato come nucleo fondante della cogenitorialità quella che identifica come "alleanza cogenitoriale", che può venire individuata nella buona comunicazione tra i partner su ciò che riguarda il figlio e il reciproco sostegno. La cogenitorialità implica che i genitori, separatamente, possano consolidare nel figlio la sensazione che sono una "unità", insegnandogli che non può averla vinta su un genitore quando l'altro è assente (McHale, 1997): questa "alleanza genitoriale" si basa fondamentalmente su un accordo tra i due partner e va negoziata in modo che non si incorra nel pericolo che ognuno dei due cambi poi arbitrariamente le regole stabilite insieme quando è solo col bambino. Cogenitorialità implica un'attenzione particolare, nei due partner, al sostegno e all'affermazione degli sforzi reciproci anche quando l'altro partner è assente.

In letteratura viene operata anche una distinzione tra ciò che si intende per alleanze coniugali e alleanze cogenitoriali: Weissman, Cohen (1985), mettono in evidenza come ci si debba riferire a queste ultime, anche se si concorda sul fatto che le difficoltà cogenitoriali possono essere più frequenti quando sono presenti anche disagi e conflitti coniugali (Talbot, McHale, 2004). In altri studi sono state rilevate relazioni significative tra l'adattamento coniugale e l'adattamento cogenitoriale (SchoppeSullivan et al., 2004), dove è stata messa in evidenza una capacità predittiva della alleanza coniugale nel periodo perinatale nei confronti della cogenitorialità, entro il terzo anno di vita del bambino. Le ricerche concordano inoltre sui collegamenti tra le dinamiche cogenitoriali e il successivo adattamento del bambino (McHale, John- 
son, Sinclair, 1999): sono stati rilevati anche collegamenti tra le dinamiche cogenitoriali nella prima infanzia e successive misure di adattamento del bambino, difficoltà cogenitoriali durante la perinatalità che sembrano predire aggressività e ansia nei bambini in età prescolare (McHale, Rasmussen, 1998) e ostilità e tensioni fra $\mathrm{i}$ partner sono peraltro apparse connesse a una maggiore disinibizione nei figli a due anni (Belsky, Putnam, Crnic, 1996).

I bambini che vivono entro famiglie con alta conflittualità coniugale sarebbero a rischio quando i problemi coniugali hanno una ricaduta sulle dinamiche cogenitoriali: è necessario pertanto rilevare separatamente i processi coniugali e cogenitoriali e non considerarli interscambiabili, perché entrambi funzionano in maniera indipendente nel predire l'adattamento del figlio (Katz, Low, 2004).

La cogenitorialità è dunque un costrutto complesso, connesso, ma anche separato dalle forme dei conflitti e disagi coniugali: è un costrutto che ha un discreto grado di stabilità nel tempo ed è collegato al funzionamento individuale di ogni partner e a quello diadico di altri sottosistemi familiari, predicendo importanti aspetti dello sviluppo del bambino nella prima infanzia.

Una valutazione della cogenitorialità (McHale, 1997) può essere effettuata attraverso una valutazione dell" "alleanza cogenitoriale" (McHale, Cowan, 1996), individuata attraverso la "solidarietà" e il "sostegno" tra i due partner che condividono la responsabilità dell'accudimento del bambino, la frequenza e l'intensità dei conflitti rispetto agli impegni, i ruoli nell'accudimento e l'impegno nell'organizzazione e gestione della vita quotidiana.

La dimensione della "solidarietà" è forse quella più importante, presenta maggiore stabilità nel tempo e si manifesta attraverso la "cooperazione intergenitoriale", individuata mediante diversi indicatori. In alcune ricerche è stata valutata attraverso quanto ogni partner approva il modo con cui l'altro esercita la propria genitorialità e il sostegno (Abidin, Brunner, 1995) e attraverso la frequenza con cui i partner si impegnano in comportamenti che stimolano il senso stesso della solidarietà (McHale, 1997). La percezione della solidarietà cogenitoriale si è rilevata connessa con la percezione di problemi comportamentali del figlio: il collegamento fra cogenitorialità e adattamento del figlio è di maggiore rilevanza rispetto alla relazione tra adattamento coniugale e adattamento del figlio. Lo stress individuale o di coppia può essere un indicatore di rischio, ma i problemi riguardanti la cogenitorialità sembrano essere il canale privilegiato attraverso cui lo stress coniugale influenza i figli.

Vengono individuati anche indicatori per la valutazione della solidarietà e del sostegno: il grado in cui le coppie si adattano reciprocamente ai diversi punti di vista sulla cura del bambino, la conferma reciproca o disconferma del partner, la disponibilità a impegnarsi in maniera collaborativa e la qualità della coordinazione quando i partner devono agire insieme nella cura del bambino (McHale, 2010). La capacità dei genitori di lavorare efficacemente insieme quando devono affrontare tensioni emotive dipende dalla possibilità di appoggiarsi su una base sicura di una loro cogenitorialità forte e supportiva. L'alleanza sarà forte quando ognuno dei due si sente sostenuto e confermato dall'altro partner. È necessario che i genitori collaborino per comporre le eventuali diversità dei loro punti di vista sulla suddivisione del lavoro di cura del bambino. 
La dimensione della “conflittualità" e dell'antagonismo (McHale et al., 2000) viene valutata operando una differenziazione tra i conflitti coniugali e quelli derivati da un disaccordo sull'allevamento del figlio. Una differenza tra le credenze dei due genitori è possibile indice di disarmonia per le differenti pratiche di allevamento.

Durante il periodo perinatale uno dei maggiori elementi di contrasto tra i genitori riguarda in particolare "l'accudimento" del bambino: un' insoddisfazione nella divisione dei compiti può alimentare sentimenti negativi nei due partner e influenzare negativamente il raggiungimento di obiettivi comuni condivisi. La divisione dei compiti nell'accudimento del bambino viene indagata attraverso il modo con cui i partner concordano sulla condivisione dei compiti e il grado di soddisfazione rispetto alle reciproche aspettative.

L'impegno e il disimpegno reciproco sono altre dimensioni che vengono considerate dallo studio di McHale: la valutazione del disimpegno e dell'investimento con i figli vengono valutate in connessione con le difficoltà dei due partner nella gestione delle proprie manifestazioni emozionali.

\subsection{Aspettative e predittività nei progetti di sostegno alle coppie}

Le aspettative prenatali prefigurano in genere la solidarietà genitoriale dopo la nascita e pertanto sono da prendere in attenta considerazione rispetto a come si svilupperanno le alleanze cogenitoriali durante il periodo perinatale.

Un pessimismo durante la gravidanza sembra precludere un'alleanza coordinata che consenta ai genitori di offrirsi una conferma reciproca: sarebbe in specifico il pessimismo della madre rispetto alla solidarietà dell'altro genitore durante la gravidanza, ma non il pessimismo del padre, a predire un ritiro maggiore da parte dell'uomo durante le negoziazioni cogenitoriali nel periodo perinatale e nei primi mesi dopo la nascita.

Le preoccupazioni prenatali rispetto alla futura cogenitorialità possono provenire sia dall'aver sperimentato tensione e disagio nella propria famiglia di origine, sia nella propria relazione di coppia. È stata individuata un'associazione significativa tra livelli di tensione coniugale e pessimismo circa le aspettative sulla propria futura famiglia. Il pessimismo prenatale non predice tuttavia un elevato conflitto cogenitoriale perinatale nei primi mesi dopo la nascita del bambino: le coppie in cui un partner mette in dubbio la propria capacità di collaborare come genitore segnalano come i partner stiano cercando di trovare dei modi per lavorare insieme in modo efficace e collaborativo. Questa aumentata sensibilità alle difficoltà di lavorare può essere anche ciò che può aver tenuto a distanza i genitori evitando lo sviluppo di una relazione genitoriale conflittuale. Le preoccupazioni prenatali dei genitori sulla solidarietà genitoriale si concretizzano in genere sulle loro difficoltà a costruire un'alleanza genitoriale supportiva e coesa, ma non prefigurano conflittualità nel periodo perinatale e nei primi mesi di vita del bimbo.

Le coppie che hanno avuto delle difficoltà nel periodo prenatale e che hanno espresso preoccupazioni sulla capacità di costruire una relazione positiva con il partner, mo- 
strano però livelli più bassi di coesione e sostegno reciproco dopo la nascita del bimbo. Non si possono tuttavia prevedere in maniera attendibile le difficoltà di una successiva coordinazione genitoriale prima dell'arrivo del bambino, anche se le prime difficoltà genitoriali possono fare prevedere quelle successive.

I dati che mettono in evidenza l'importanza di questi aspetti di previsione sono utilizzati per i progetti di sostegno alle coppie quando stanno per diventare genitori. Le aspettative familiari prenatali e i pattern relazionali della coppia sono in genere abbastanza buoni pronostici di come sarà lo sviluppo successivo dopo la nascita del bambino. In un progetto di prevenzione primaria è utile individuare allora quali siano le aspettative dei genitori, le problematiche importanti da indagare, e quali possano essere le tematiche maggiormente significative e formative per la coppia che si appresta alla genitorialità.

Utilizzando la teoria dell'attaccamento è possibile conoscere come i genitori abbiano vissuto le proprie esperienze di accudimento nella famiglia di origine: queste influenzano la qualità della relazione che svilupperanno con i propri figli (Talbot et al., 2006). Secondo i dati della ricerca di McHale (2010), il conflitto cogenitoriale sarebbe predetto da fattori inerenti le configurazioni dell'attaccamento. Le madri che hanno presentato pattern di attaccamento insicuro verso i propri genitori, durante la propria infanzia manifesterebbero maggiori probabilità di sviluppare conflittualità cogenitoriali quando il bimbo è nato, nel periodo perinatale. I pattern di attaccamento $\mathrm{e}$ in particolare l'insicurezza materna devono essere pertanto valutati attentamente durante il periodo prenatale e considerati come un fattore potenziale di rischio, non solo di attaccamento insicuro da parte del bimbo, ma anche di manifestazioni di conflitti cogenitoriali (Talbot et al., 2006). I padri che manifestano un pattern di attaccamento insicuro hanno minori probabilità delle madri di venire coinvolti in conflitti cogenitoriali nel periodo perinatale, ma presentano maggiori comportamenti di ritiro a tre mesi dalla nascita del bambino. In coppie che sperimentano tensioni coniugali, in cui i genitori sono piuttosto pessimisti sulle proprie capacità di alleanza genitoriale, esiste una maggiore possibilità che il processo genitoriale sia caratterizzato da bassi livelli di solidarietà e sostegno alla nascita del bambino.

Le ricerche che indagano le dinamiche genitoriali delle famiglie di origine e le aspettative nei confronti delle proprie future famiglie hanno permesso di rilevare che non sempre i genitori che riferiscono problemi di alleanza genitoriale da parte dei propri genitori sono esposti a tale rischio nella propria famiglia, mentre sarebbero maggiormente esposti a rischio i genitori che hanno espresso aspettative negative rispetto alla propria futura famiglia. Queste ricerche evidenziano problemi di coppia nel periodo perinatale (McHale, Kazali, Rotman, 2004), ma anche difficoltà nei periodi successivi (McHale, Rotman, 2007): i ricordi sulla carenza di cure ricevute nella propria infanzia sono meno predittivi delle preoccupazioni e previsioni negative che $i$ genitori esprimono riguardo alla loro futura famiglia. I problemi con la famiglia di origine non sono irrilevanti, ma costituiscono una base a cui i genitori possono fare riferimento per le previsioni nei confronti della propria famiglia, nonché per attivarsi consapevolmente per non sviluppare i propri modelli genitoriali negativi nella loro nuova famiglia.

Quando comunque la coppia prevede di non essere in grado di lavorare bene in- 
sieme nella cogenitorialità, vi sono alte percentuali di rischio. Nella ricerca di McHale (2010) viene data maggiore importanza alle situazioni della vita attuale dei genitori e alla qualità della loro relazione quali indicatori predittivi. I partner della coppia che hanno attraversato esperienze difficili nella loro infanzia, ma che hanno trovato il modo di parlarne, così come dei propri sogni e dei desideri riguardanti la propria futura famiglia, si troverebbero in una posizione migliore per affrontare quelle paure e preoccupazioni che la propria famiglia di origine ha suscitato in loro. Queste tematiche possono evocare ansia, ma consentire ai partner di sperimentare il sostegno e il contatto reciproco: se i problemi per la genitorialità possono venire espressi nello spazio della coppia, si pongono le basi per la cogenitorialità.

La valutazione dell'alleanza cogenitoriale può basarsi, oltre che sulle osservazioni dei genitori e del figlio, su interviste e questionari che possono essere usati come stimolo per sollecitare i genitori a parlare del grado di sostegno che sentono di ricevere dal loro partner genitoriale o della divergenza che esiste tra loro sul modo di considerare la genitorialità. Nessun indicatore tuttavia è capace di descrivere tutto quello che sarebbe importante rilevare e quindi sono auspicabili valutazioni molteplici. Insomma, come si evince dalla breve rassegna che abbiamo citato, le ricerche sono molteplici, spesso differentemente centrate, altrettanto spesso effettuate con strumenti diversi: si spiegano così aspetti di eventuali frammentarietà o di scarsa concordanza, se non talvolta di contraddizioni. Tutto ciò dimostra la complessità dei fattori che si intrecciano nel generare problemi di cogenitorialità e il loro differente aspetto, nonché possibilità di misura, a seconda di un più ampio e generale contesto: questo dovrebbe indurre la massima attenzione e prudenza nell'impostare programmi di sostegno alle coppie.

Secondo le rilevazioni effettuate da McHale prima della nascita del figlio, i genitori raramente parlano insieme del modo in cui si occuperanno del bambino che sta per arrivare: è importante pertanto che in un programma di prevenzione si chieda a ogni genitore di esprimere le proprie idee sulla genitorialità e fare previsioni su quali pensa siano quelle del proprio partner. È necessario pertanto lavorare sul sistema di "previsioni" che i genitori hanno, sollecitandoli a esprimerle e a fare inferenze sui propri e altrui comportamenti futuri: questo servirà a innescare una riflessione tra $\mathrm{i}$ genitori (Schultz, Cowan, Cowan, 2006) che potrà consentire un chiarimento. Le opinioni prenatali dei genitori rispetto a come vorrebbero fosse il loro bambino hanno un valore prognostico notevole: discutere delle differenze di opinioni durante la gravidanza non impedisce che il conflitto e le convinzioni divergenti possano riemergere con la nascita del figlio, tuttavia il fatto che la coppia si interroghi e riconosca in modo consapevole di avere punti di vista diversi è importante per muoversi in una posizione genitoriale che tenga conto delle rispettive opinioni.

All'arrivo del bambino sarebbe necessario che la coppia costruisse un'alleanza che permetta di affrontare le varie difficoltà: depressione post partum, frustrazioni per la perdita d'indipendenza e di intimità, irritazione per interferenze di genitori e suoceri. Quando i rapporti diventano conflittuali ne risentono le relazioni, e le dinamiche cogenitoriali si spostano sull'antagonismo e la conflittualità, con il bambino che diventa il punto focale di riferimento, e così si trova inserito nelle dinamiche disfunzionali della coppia. 
La ricerca di McHale focalizza un dato interessante: nelle famiglie in cui entrambi i genitori lavorano (Mc Hale, 2010) si riscontrerebbe una cogenitorialità meglio coordinata. Il funzionamento cogenitoriale sembra essere molto positivo quando i genitori lavorano entrambi e il bambino deve essere accudito durante il giorno da parenti o altre persone estranee alla famiglia; questo contribuirebbe a rafforzare le alleanze cogenitoriali. Nelle famiglie in cui la madre lavora, si sono rilevati pattern di attaccamento sicuro nel bimbo nei confronti del padre (Chase-Landsale, Owev, 1987): il padre viene maggiormente coinvolto nella cura dei figli (Crouter, Perry-Jenkins, Huston, 1987), è più informato anche delle loro attività scolastiche (Crouter, Helms-Erikson, Updegraff, 1999), tende a confermare gli sforzi della madre; i genitori, lavorando entrambi, si impegnano maggiormente in maniera collaborativa (Fish, McHale, 2001). Le famiglie in cui i genitori lavorano cercano con maggiore intensità di confermare e sostenere gli sforzi reciproci. Si è rilevata inoltre un'intensificazione dell'interazione madre-bambino nei momenti in cui la diade si ritrova insieme, alla fine della giornata, con un miglioramento della qualità della relazione stessa (Zaslow et al., 1986, 1989).

Un altro dato interessante è come variano, durante il primo anno, le dinamiche cogenitoriali in funzione delle esperienze di cura giornaliera dedicate ai bambini (McHale, 2010): le famiglie che si avvalevano di servizi come nidi, baby-sitter, familiari sono state divise in due gruppi a seconda del numero di ore a cui bambini venivano affidati a estranei. L'investimento madre-figlio e la discrepanza rispetto al coinvolgimento del padre con il bambino sono risultati maggiori nelle famiglie in cui i bambini venivano affidati per un numero minore di ore ai servizi. Questo dato ha posto la questione su quale sia la situazione migliore di cura giornaliera per il bambino: le famiglie che affidano per un maggior numero di ore i bambini ai servizi si impegnano notevolmente per stare insieme più tempo alla fine della giornata e passare in famiglia il massimo tempo possibile. Favoriscono in tal modo calore familiare e conferma genitoriale e il padre sembra intervenire a sostenere maggiormente la madre. Le madri che invece stanno con $\mathrm{i}$ bambini più spesso durante la giornata, svilupperebbero abitudini molto più consolidate con il bambino rispetto ai padri e questo spiegherebbe le discrepanze riscontrate rispetto al coinvolgimento di entrambi i genitori nelle interazioni familiari. Le madri che stanno maggiormente a casa assumerebbero quello che viene chiamato il "ruolo del guardiano" (De Luccie, 1995), escludendo i padri.

Questi dati sarebbero incoraggianti (Fish, McHale, 2001) in una società in cui entrambi i genitori sono sempre più impegnati in attività lavorative extra domestiche, e sarebbe un dato confortante per le coppie di genitori che prevedono di proseguire l'attività lavorativa dopo la nascita del bimbo.

Le situazioni conflittuali fanno parte dell'esperienza delle famiglie e il modo in cui vengono risolte modella nel corso del tempo le capacità di regolazione stessa del bambino. L'alleanza cogenitoriale è un fattore protettivo con effetti positivi sullo sviluppo del bambino (Floyd, Gilliom, Costigan, 1998; Margolin, Gordis, John, 2001). Fornire un ambiente affidabile che dia regole equilibrate e limiti adeguati consentendo un'espressione emotiva, aiuta il bimbo a esprimere la propria indipendenza e autonomia adeguate all'età e può fornirgli esperienze congrue allo sviluppo e alla cre- 
scita. Se i genitori hanno obiettivi contrastanti i bambini apprendono che le regole sono inaffidabili e arbitrarie e che possono rispettarle in modo diverso a seconda della circostanza.

Un paradigma sperimentale di ricerca (Hirshberg, 1990) ha fornito evidenze sperimentali sullo stato di disagio in cui si viene a trovare un bambino quando i genitori gli inviano messaggi contemporaneamente contrastanti: nella situazione sperimentale un bambino di un anno viene esposto a uno stimolo potenzialmente ansiogeno, paragonabile a un precipizio visivo che deve attraversare, mentre i genitori gli inviano contemporanei segnali contrastanti, mettendo il bambino di fronte a un dilemma difficilmente risolvibile. Egli manifesterà spesso un comportamento disorganizzato e di grande stress. Queste ricerche evidenziano come il bambino, esposto dai suoi genitori a posizioni contrastanti, in cui nessuna decisione è positiva, diventi dominato da insicurezza e disorganizzazione.

Padri e madri cresciuti in famiglie in cui i genitori avevano difficoltà e alimentavano insicurezza nei figli hanno difficoltà a mettersi in discussione prima di diventare a loro volta genitori. Avendo alle spalle un modello conflittuale di genitorialità, dovrebbero poter elaborare le proprie esperienze angoscianti e difficili, ma ciò spesso non avviene. In molte situazioni i partner sono consapevoli di ciò, ma si sentono semplicemente incapaci di cambiare: occorre pertanto poter provvedere a un adeguato aiuto.

Le ricerche evidenziano come nella coppia sono in genere i padri a celare maggiormente le proprie frustrazioni e preoccupazioni, forse cercando di farsene carico per proteggere la propria compagna (Wile, 1988): ogni partner pertanto dovrebbe essere aiutato, a diventare consapevole del suo stile genitoriale e di come questo possa accordarsi o meno a quello dell'altro. Non è possibile prevedere come manifestazioni del disagio personale possano essere condivise dal proprio partner e come preoccupazioni relazionali possano venire decodificate, tuttavia i problemi genitoriali spesso nascono proprio dalla mancanza di questi confronti; molto spesso i genitori dichiarano di non aver mai parlato con il partner delle proprie preoccupazioni con sincerità (McHale, 1995).

\section{Bibliografia}

Abidin RR, Brunner JF (1995) Development of a parenting alliance inventory. Journal of Clinical Child Psychology 24:31-40

Ammaniti M (1992) La gravidanza tra fantasia e realtà. Il Pensiero Scientifico, Roma

Ammaniti M, Candelori C, Pola M, Tambelli R (1995) Maternità e gravidanza. Studio delle rappresentazioni materne. Cortina, Milano

Ammaniti M Stern DN (1992) Attaccamento e psicoanalisi. Laterza, Bari

Badinter E (1981) L'amore in più. Storia dell'amore materno. Longanesi, Milano

Baldoni F (2005) Funzione paterna e attaccamento di coppia: l'importanza di una base sicura. In Bertozzi N, Hamon C (a cura di) Padri e paternità. Junior, Bergamo

Baruffi L (1979) Il desiderio di maternità. Boringhieri, Torino

Belsky J, Putnam S, Crnic K (1996) Coparenting, parenting and early emotional development. New Directions for Child Development 74:45-55

Benedek T (1958) Parenthood as a development phase: a contribution to the libido theory. Journal of the American Psychoanalytic Association 7:389-417 
Bibring GL (1959) Some considerations of the psychological process in pregnancy. The Psychoanalytic Study of the Child 16:113-121

Bick E (1964) Nota sull'osservazione del lattante nell'addestramento psicoanalitico. Trad.it. Bonaminio V, Iaccarino B (a cura di) L'osservazione diretta del bambino. Boringhieri, Torino, 1984

Bion WR (1962) Learning from experience. Heinemann, London. Tr.it. Apprendere dall'esperienza. Armando, Roma, 1972

Bowlby J (1979) Costruzione e rottura dei legami affettivi. Cortina, Milano, 1982

Bowlby J (1988) Una base sicura. Cortina, Milano, 1989

Brazelton TB, Cramer (1991) Il primo legame. Frassinelli, MilanoBrockington I (2004) Post-partum psychiatric disorders. Lancet 363:303-310

Bruner JS, Ross G, Wood D (1976) The role of tutoring in problem solving. Journal of Child Psychology and Psychiatry 17, Pergamon Press

Bydlowski M (1989) Desiderio di un bambino, desiderio di gravidanza, evoluzione delle pratiche della procreazione. Trad.it. Lebovici S. Weil-Halpern F, Psicopatologia della prima infanzia. Boringhieri, Torino

Bydlowski M (1991) La transparence psychique de la grossesse. Etudes freudiennes, 135-142

Bydlowski M (1997) Il debito di vita. Itinerario psicoanalitico della maternità. Quattroventi, Urbino, 2000

Bydlowski M, Raoul-Duval A (1978) Un avatar psychique méconnu de la puerperalité: la névrose traumatique post obstétricale. Perspectives Psychiatriques 4:321-328

Capello C, Vacchino R (1985) Sessualità femminile e istituzioni sociali. E.T.S., Pisa

Cena L (2005a) Costruire una mente: la competenza comunicativa primaria della gestante. Nascere 95:20-33

Cena L (2005b) Competenza comunicativa primaria e movimenti fetali. Nascere 96:24-34

Cena L, Imbasciati A (2007) Il desiderio di generare. In: Imbasciati A, Dabrassi F, Cena L (a cura di) Psicologia clinica perinatale. Piccin, Padova

Cena L, Imbasciati A, Baldoni F (2010) La relazione genitore-bambino. Springer, Milano

Chase-Lansdale PL, Owen MT (1987) Maternal employment in a family context: Effects on infantmother and infant-father attachments. Child Development 58:1505-1512

Crittenden PM (2008) Il Modello Dinamico Maturativo dell'Attaccamento. Cortina, Milano

Crittenden PM, Landini A (2010) Un modello evoluzionistico della teoria dell'attaccamento: l'intervento clinico. In Cena L, Imbasciati A, Baldoni F (2010) La relazione genitore-bambino, Springer, Milano

Crouter A C, Perry-Jenkins M, Huston T L, McHale S M (1987) Processes underlying father involvement in dual-earner and single-earner families. Developmental Psychology 23:431440

Crouter A, Helms-Erikson H, Updergraff K (1999) Conditions underlying parents' knowledge about children's daily lives in middle childhood: between and within family comparisons. Child Development 70:246-259

Darchis E (2009) L'instaurazione della genitorialità e le sue vicissitudini. In Zurlo MC, (a cura di) Percorsi della filiazione. Angeli, Milano

De Luccie MF (1995) Mothers as gatekeepers: A model of maternal mediators of father involvement. journal of Genetic Psychology 152:225-238

Deutsch H (1945) Psicologia della donna. Vol. I (1945) Vol. II (1946), Boringhieri, Torino, 1971

Erikson EH (1950) Infanzia e società. Armando, Roma

Feeney BC, Collins NL (2004) Processi interpersonali di accudimento. Porto di salvezza e base sicura nell'attaccamento adulto. Trad it. Rholes WS, Simpson JA (a cura di) Teoria e ricerca nell'attaccamento adulto. Cortina, Milano, 2007

Fish K, McHale J (2001) Parental employment, coparenting, and family interaction at one year. Poster presented at the Society for Research in Child Development

Floyd FJ, Gilliom IA, Costigan CL (1998) Marriage and the parenting alliance: longitudinal prediction of change in parenting perceptions and behaviors. Child Development 69:1461-1479

Fonagy P (2001) Psicoanalisi e teoria dell'attaccamento. Cortina, Milano

Fraiberg SH, Adelson E, Shapiro V (1975) I fantasmi nella stanza dei bambini. Un approccio psi- 
coanalitico ai problemi posti da relazioni disturbate madre-bambino. Trad.it. Fraiberg SH. Il Sostegno allo sviluppo. Cortina, Milano, 1999

Freud S (1915) Metapsicologia.In Opere di Sigmund Freud Vol VIII. Boringhieri, Torino

Freud S (1926) L'analisi dei non medici. Opere, Vol X. Boringhieri, Torino

Freud S (1931), Sessualità femminile. In Opere di Sigmund Freud Vol XI. Boringhieri, Torino

Ghilardi A, Imbasciati A (1989) Il concetto di istinto. Neurologia, Psichiatia e Scienze Umane 6:10351056

Giannakoulas A, Giannotti A (1985) Il setting con la coppia genitoriale. In Il setting. L'approccio relazionale in Neuropsichiatria infantile. Borla, Roma

Golse B (1995) Le bebé, l'hôpital, les équipes soignantes et les psys. In: Ben Soussan P et al (a cura di) Le Bébé a l'Hôpital. Syros, Paris

Hirshberg LM (1990) When infant look to their parents: Twelve month-olds response to conflicting parental emotional signals. Child Development 61:1187-1191

Imbasciati A (1990) La donna e la bambina. Angeli, Milano

Imbasciati A (2005) La sessualità e la teoria energetico-pulsionale. Freud e le conclusioni sbagliate di un percorso geniale. Angeli, Milano

Imbasciati A (2007) Neurosciences et Psychanalyse: pour une nouvelle metapsychologie,

Revue Française de Psychanalyse LXXI, 2:455-477

Imbasciati A (2008) Le cure materne: matrice dello sviluppo del futuro individuo. Nascere XXXV:4-14

Imbasciati A (2010) Towards new metapsychologies Psychoanalytic Review 1:44-46

Imbasciati A (2011a) Perché la sessualità. Piccin, Padova

Imbasciati A (2011b) The meaning of a metapsychology. Journal Academy Psychoanal and Dynamic Psychiatric

Imbasciati A, Buizza C (2011) l'emozione sessuale. Psicoanalisi e neuropsicofisiologia. Liguori, Napoli

Imbasciati A, Cena L (1987) Uno studio sulla femminilità attraverso l'analisi del mito. In: Il laboratorio e la Città. Lo Psicologo professionista e ricercatore. XXI Congresso degli Psicologi Italiani, Atti, 685-691

Imbasciati A, Cena L (1988) La donna nel mito greco. Neurologia Psichiatria e Scienze Umane. Vol VIII, 3:435-455

Imbasciati A, Cena L (2007 a) Psicosomatica della gravidanza e del parto. In: Imbasciati A, Dabrasssi F, Cena L (2007) Psicologia Clinica Perinatale. Piccin, Padova

Imbasciati A, Cena L (2007b) La comunicazione gestante-feto. In: Imbasciati A, Dabrasssi F, Cena L (2007) Psicologia Clinica Perinatale. Piccin, Padova

Imbasciati A, Cena L (2007c) Sviluppo del bimbo e sviluppo della madre. In: Imbasciati A, Dabrasssi F, Cena L (2007) Psicologia Clinica Perinatale. Piccin, Padova

Imbasciati A, Cena L (2011) Sessualità, generatività, genitorialità. In Imbasciati A, Buizza C, L'emozione sessuale. Liguori, Napoli

Imbasciati A, Dabrasssi F, Cena L (2007) Psicologia Clinica Perinatale. Piccin, Padova

Imbasciati A, Dabrasssi F, Cena L (2011) Psicologia Clinica Perinatale per lo Sviluppo del Futuro Individuo. Un uomo transgenerazionale. Express, Torino

Imbasciati A, Ghilardi A (1990) L'istinto nelle scienze: necessità e storia di un concetto irresolubile. Rivista di sessuologia 2:101-106

Kaes R (1982) Il sostenimento gruppale dello psichismo individuale: alcune conseguenze teoriche riguardo I concetti di individuo e di gruppo. Quaderni di Psicoterapia di Gruppo 1:57-73

Katz LF, Low SM (2004) Marital violence, co-parenting and family level processes in relation to children's adjustment. Journal of Family Psychology 18:372-382

Klain M (1932) La psicoanalisi dei bambini. Martinelli, Firenze

Kraisler L, Cramer B (1981) Les bases cliniques de la psychiatrie du nourrisson. Psychiatrie de l'enfant 24:223-263

Lebovici S (1989) I legami intergenerazionali. Le interazioni fantasmatiche. Trad. It. Lebovici S, Weio-Halpern F, Psicopatologia della prima infanzia. Boringhieri, Torino

Lebovici S (1994) Les interactions fantsmatiques. Revue de Medicine Psychosomatique 37/38:39-50

Manfredi P, Imbasciati A (2004) Il feto ci ascolta e impara. Borla, Roma 
Margolin G, Gordis FB, John RS (2001) Coparenting: a link between marital conflict and parenting in two parent families. Journal of Family Psychology 15:3-21

McHale JP (1995) Coparenting and triadic interactions during infancy: The roles of marital distress and child gender. Developmental Psychology 31:985-996

McHale JP (1997) Overt and covert coparenting processes in the family. Family Process 36:183201

McHale JP (2010) La sfida della cogenitorialità. Cortina, Milano

McHale JP, Cowan PA (1996) Understanding How Family-level Dynamics Affect Children's Development: Studies of Two-parent Families. Jossey-Bass, San Francisco

McHale JP et al (2004) The transition to coparenthood: Parent's prebirth expectations and early coparental adjustement at 3 months postpartum. Development and Psychopathology 16:711-733

McHale JP, Rotman T (2007) Is seeing believing? Expectant parents'outlooks on coparenting and later coparenting solidarity. Infant Behavior and Development 30:63-81

McHale JP, Kuersten-Hogan R, Lauretti A, Rasmussen JL (2000) Parental reports of coparenting and observed coparenting behavior during the toddler period. Journal of Family Psychology 14:220-236

McHale JP, Johnson D, Sinclair R (1999) Family dinamics prescoolers family representations and prescool peer relationships. Early Education and Development 10:373-401

McHale JP, Rasmussen JL (1998) Coparental and family group-level dynamics during infancy: early family precursors of child and family functioning during preschool. Developmental and Psychopathology 10:39-59

Minuchin S (1974) Famiglia e terapia della famiglia. Astrolabio, Roma, 1977

Moro MR, Nathan T, Rabain J, Stork H, Si Ahmed D (1989) Il lattante nel suo universo culturale Trad it. Lebovici S, Weil-Halpern F, Psicopatologia della prima infanzia. Boringhieri, Torino, 1994

Palacio Espasa F (1991) Fantasie dei genitori e psicopatologia del bambino. In: Società di Neuropsichiatria infantile (a cura di) Fantasie dei genitori e psicopatologia dei figli. Borla, Roma

Pines D (1972) Pregnancy and motherhood: interaction between fantasy and reality. British Journal of Medical Psychology 45:333-343

Pines D (1982) The revelance of early psychic development to pregnancy and abortion. International Journal of Psychoanalysis 63:311-319

Scabini E (1985) L'organizzazione famiglia tra crisi e sviluppo. Angeli, Milano

Scabini E (1995) Psicologia sociale della famiglia. Boringhieri, Torino

Schoppe-Sullivan S.J, Mangelsdorf S.C, Frosch C.A, Mc Hale JP (2004) Associations between coparenting and marital behavior from infancy and the preschool years. Journal of Family Psycho$\log 18: 194-207$

Shaffer R (1980) Developing child. In: Bruner J Cole N, LLyod B (a cura di) Maternità. Armando, Roma

Soifer R (1985) Psicologia della gravidanza, parto e puerperio. Borla, Roma

Schulz MS, Cowan PA, Cowan CP (2006) Promoting healthy beginnings: A randomized controlled trial of a preventive intervention to preserve marital quality during the transition to parenthood. Journal of Clinical and Consulting Psychology 74:20-31

Stern DN (1985) Il mondo interpersonale del bambino. Boringhieri Torino, 1987

Stern DN (1995) La costellazione materna. Boringhieri, Torino

Stoleru S (1998) De l'arrêt de la contraception aux premiers mois postnatals-. Les premières étapes de la parentalité adulte. In: Mazet P, Lebovici S (a cura di) Psychiatrie périnatale. PUF, Paris

Stoleru S, Morales-Huet M (1989) Processus de recherche et processus psychothérapique. In L'évaluation des interactions précoces. Eshel, Paris

Talbot J, Elliston D et al (2006) Do early coparenting dynamics benefit from coherent states of mind with respect to attachment? Infant Mental Health Journal 27:43

Talbot JA, McHale JP (2004) Individual parental adjustment moderates the relationship between marital and coparenting quality. Journal of Adult Develpment 11:191-205

Vegetti Finzi S (1990) Il bambino della notte. Mondadori, Milano

Vegetti Finzi S (1997) Volere un figlio. La nuova maternità tra natura e scienza. Mondadori, Milano 
2 Vygoskij LS (1990) Pensiero e linguaggio. Ricerche psicologiche. Laterza, Bari

Weissman HS, Cohen SR(1985) The parenting alliance and adolescence. Adolescent Psychiatric $12: 24-45$

Wile DB (1988) In search of the curative principle in couples therapy. Journal of Family Psychology $2: 24-27$

Winnicott DW (1956) La preoccupazione materna primaria. In Winnicott DW (1958) Dalla pediatria alla psicoanalisi. Martinelli, Firenze, 1991

Winnicott DW (1958) Dalla pediatria alla psicoanalisi. Martinelli, Firenze, 1991

Winnicott DW (1965) Sviluppo affettivo e ambiente. Armando Editore, Roma, 1970

Zaslow MJ, Pedersen FA, Suwalsky T, Rabinovich B (1986) Fathering during the infancy period: implications of the mothers employement role. Infant Mental Healt Journal 7:225-234

Zaslow MJ, Pedersen FA, Suwalsky JT, Rabinovich B (1989) Maternal employment and parent-infant interaction at one year. Early Chilhood Research Quarterly 4:459-478

Zurlo MC (2009) Percorsi della filiazione. Angeli, Milano 